\title{
A Mesoscopic Electromechanical Theory of Ferroelectric Films and Ceramics
}

\author{
Jiangyu $\mathrm{Li}^{*}$ and Kaushik Bhattacharya ${ }^{\dagger}$ \\ *Department of Engineering Mechanics, W317.5 Nebraska Hall, University of Nebraska-Lincoln, \\ Lincoln, NE 68588-0526 \\ ${ }^{\dagger}$ Division of Engineering \& Applied Science, Mail Stop 104-44, California Institute of Technology, \\ Pasadena, CA 91125
}

\begin{abstract}
We present a multi-scale modelling framework to predict the effective electromechanical behavior of ferroelectric ceramics and thin films. This paper specifically focuses on the mesoscopic scale and models the effects of domains and domain switching taking into account intergranular constraints. Starting from the properties of the single crystal and the pre-poling granular texture, the theory predicts the domain patterns, the post-poling texture, the saturation polarization, saturation strain and the electromechanical moduli. We demonstrate remarkable agreement with experimental data. The theory also explains the superior electromechanical property of PZT at the morphotropic phase boundary. The paper concludes with the application of the theory to predict the optimal texture for enhanced electromechanical coupling factors and high-strain actuation in selected materials.
\end{abstract}

\section{INTRODUCTION}

It has recently been recognized that it is possible to obtain large actuation strains in ferroelectric single crystals through polarization rotation $[1,2]$, or by domain switching as a result of applied electric field and mechanical stress [3, 4]. However, large strains cannot usually be obtained in a polycrystalline ferroelectrics due to the incompatibility between neighboring grains. So the identification of the optimal texture of polycrystals for high-strain actuation is a worthwhile challenge. A related problem is the poling of the piezoelectric ceramic PZT, which is a solid solution of lead titanate and lead zirconate. The titanium rich compositions where the material is tetragonal, and the zirconium rich compositions where the material is rhombohedral are both very difficult to pole, while the material is dramatically easy to pole at the 'morphotropic phase boundary' [5]. An understanding of this difference is also desirable.

Polycrystalline ferroelectrics consist of numerous grains (regions of continuous lattice with uniform pseudo cubic orientation), which in turn contain domain (regions of uniform polarization amongst those allowed by the orientation of the grains). Processing methodology affects the orientation distribution of the grains while poling affects the orientation distribution of the domains. We used texture to mean the orientation distribution of both grains and domains.

This work examines the macroscopic behaviors of ferroelectric solids in terms of the crystal systems and texture, using the homogenization theory and energy minimization following the framework used by Bhattacharya and Kohn [6] to study polycrystalline shape-memory alloys and extended to ferroelectrics by Li and Bhattacharya [7].

CP626, Fundamental Physics of Ferroelectrics 2002, edited by R. E. Cohen

(C) 2002 American Institute of Phvsics 0-7354-0079-2/02/\$19.00

224 
The post-poling texture, spontaneous strain and polarization, and the effective electromechanical moduli for polycrystalline ferroelectrics are characterized, and remarkable agreement with experimental data has been obtained. The theory also identifies the optimal pre-poling texture for high-actuation strain and enhanced electromechanical coupling for polycrystals, and explains the optimal electro-mechanical property of PZT at the morphotropic phase boundary. This paper reports on the progress since [8], and the reader is referred to $\mathrm{Li}$ and Bhattacharya [7] for details.

\section{THEORETICAL FRAMEWORK}

\section{The Effective Electroelastic Energy}

Consider a polycrystalline ferroelectric $\Omega$ subject to an applied mechanical load (traction) $\mathbf{t}_{0}$ on part of its boundary $\partial_{2} \Omega$ and external applied electric field $\mathbf{E}_{0}$. The displacement $\mathbf{u}$ and polarization $\mathbf{p}$ of the ferroelectric are those that minimize the potential energy

$$
\int_{\Omega}\left\{\frac{1}{2} \nabla \mathbf{p} \cdot \mathbf{A} \nabla \mathbf{p}+W(\mathbf{x}, \mathbf{e}[\mathbf{u}], \mathbf{p})-\mathbf{E}_{0} \cdot \mathbf{p}\right\} d \mathbf{x}-\int_{\partial_{2} \Omega} \mathbf{t}_{0} \cdot \mathbf{u} d S+\int_{\mathbb{R}^{3}} \frac{\varepsilon_{0}}{2}|\nabla \phi|^{2} d \mathbf{x} .
$$

Above, $\mathbf{A}$ is a positive-definite matrix so that the first term above penalizes sharp changes in the polarization, and may be regarded as the energetic cost of forming domain walls. The second term $W$ is the stored energy density (Landau energy density) which depends on the state variables or order parameters, strain $\mathbf{e}$ and polarization $\mathbf{p}$, and also explicitly on position $\mathbf{x}$ in polycrystals. $W$ encodes all the crystallographic and texture information. The third term is the potential associated with the applied electric field, while the fourth term is the potential energy associated with the applied mechanical load. The final term is the electrostatic field energy that is generated by the polarization distribution. For any polarization distribution, the electrostatic potential $\phi$ is determined by solving Maxwell equation in all space subject to appropriate boundary conditions. Thus, this last term is non-local. This potential is essentially one used in the classical Landau-GinzburgDevonshire theory, except the electrostatic contribution to energy is accounted for with some care.

Minimizing (1) over all possible displacements $\mathbf{u}$ and polarization $\mathbf{p}$ leads to the formation of domains in each grain [4]. If $|\mathbf{A}|$ is very small (specifically if the squareroot of the largest eigenvalue of $\mathbf{A}$ is small compared to the grain size), then the domains are much smaller than the grains and we may set $\mathbf{A}=\mathbf{0}$ in (1) in order to determine the nature of the domain patterns and the effective energy [9]. Henceforth, we make this assumption.

The functional (1) (with $\mathbf{A}=\mathbf{0}$ ) describes all the details of the domain pattern in each grain, and thus is rather difficult to understand. It would be very useful to obtain a functional that directly captures the effective macroscopic behavior of all the domains and grains without describing all the details. Suppose the texture of polycrystal is periodic with period $\varepsilon>0$. Then, as $\varepsilon \rightarrow 0$, the effective behavior of the polycrystal 
is described by the effective functional [7]

$$
\int_{\Omega}\left\{\bar{W}(\mathbf{e}[\mathbf{u}], \mathbf{p})-\mathbf{E}_{0} \cdot \mathbf{p}\right\} d \mathbf{x}-\int_{\partial_{2} \Omega} \mathbf{t}_{0} \cdot \mathbf{u} d S+\int_{\mathbb{R}^{3}} \frac{\varepsilon_{0}}{2}|\nabla \phi|^{2} d \mathbf{x} .
$$

Note that we have replaced $W$ in (1) with $\bar{W}$ which does not explicitly depend on position $\mathbf{x} . \bar{W}(\mathbf{e}, \mathbf{p})$ is the average stored energy in the ferroelectric ceramic with average polarization $\mathbf{p}$ and average strain $\mathbf{e}$ after taking into account the domain patterns and the grains. We therefore call it the effective energy density, and is characterized by the variational formula,

$$
\bar{W}(\mathbf{e}, \mathbf{p})=\inf _{k} \inf _{<\mathbf{e}^{\prime}>=0<\mathbf{p}^{\prime}>=0} \inf _{|k Q|} \frac{1}{\mid k Q} \int_{k Q}\left\{W\left(\mathbf{y}, \mathbf{e}+\nabla \mathbf{u}^{\prime}, \mathbf{p}+\mathbf{p}^{\prime}\right)+\frac{\varepsilon_{0}}{2}\left|\nabla \phi^{\prime}\right|^{2}\right\} d \mathbf{y} .
$$

We can evaluate this in two steps. Heuristically, we can first take the effect of domain patterns into account and define an effective energy density of the single crystal $\tilde{W}$. We can then average over the grains to obtain $\bar{W}$. Mathematically, we first apply the formula (3) but with y fixed in the argument of $W$ to obtain $\tilde{W}$. This is the effective energy of a single crystal. We then evaluate (3) with $W$ replaced with $\tilde{W}$.

This is unfortunately too complicated to perform explicitly. We can do the first step in some important cases, but we use bounds to estimate the second. Of particular interest is those states that make $\bar{W}=0$. These are all possible remnant polarizations and remnant strains of ferroelectric ceramic, and the extreme points of this set are the saturation polarizations and saturation strains. We call our estimate of this set the Taylor estimate $\mathbb{Z}^{T}$. We refer the reader to Li and Bhattacharya [7] for details.

\section{Texture and Effective Moduli}

The Taylor estimate can also provide valuable information on their texture. The orientation of grains in a ferroelectric ceramic could be described by $\theta_{g}$, the angle between the global poling axis of ceramic and the local crystallographical axis of grains. Within each grain there are numerous domains whose orientation can be described by $\theta_{d}\left(\theta_{g}\right)$, the angle between the polarization direction of domains and the poling direction of ceramic, which depends on the grain's orientation $\theta_{g}$ through crystallographic constraints. The statistical distribution of $\theta_{g}$ and $\theta_{d}$ are described by the orientation distribution function (ODF) $G\left(\theta_{g}\right)$ and $D\left(\theta_{d}\right)$, which gives the probability of finding a grain or a domain in a particular orientation. In the as-processed states, both grains and domains are randomly

oriented, so that $G$ and $D$ are uniform and independent of $\theta_{g}$ and $\theta_{d}$, and the ceramic is isotropic and non-piezoelectric. The poling process, where a high electric field is applied to ceramics at elevated temperature, tends to realign the polarization direction of domains as closely as possible to the applied field, thus changes the orientation distribution of domains, $D\left(\theta_{d}\right)$, but does not change the orientation distribution of grains, $G\left(\theta_{g}\right)$. The change in orientation distribution of domains leads to macroscopic piezoelectricity in ceramics, which we analyze here. 
The actual orientation distribution of domains in a poled ferroelectric ceramic is rather difficult to determine, but could be fairly well approximated by the Taylor estimate. In other words, saturation state in ferroelectric ceramics is characterized by the Taylor estimate as $\left(\mathbf{e}^{s}, \mathbf{p}^{s}\right)$, which is also the saturation strain and polarization in all grains. This leads to

$$
\sum_{i=1, n} f_{i}\left(\boldsymbol{\theta}_{g}\right) \mathbf{e}_{i}\left(\boldsymbol{\theta}_{g}\right)=\mathbf{e}^{s}, \quad \sum_{i=1, n} f_{i}\left(\boldsymbol{\theta}_{g}\right) \mathbf{p}_{i}\left(\boldsymbol{\theta}_{g}\right)=\mathbf{p}^{s},
$$

where $f_{i}, \mathbf{e}_{i}, \mathbf{p}_{i}$ are the volume fraction, transformation strain, and transformation polarization of variant $i$ in grains at orientation $\theta_{g}$, respectively. The orientation of variant $i, \theta_{d}^{i}$, could be uniquely determined from crystallography. The equation can be solved to determine $f_{i}\left(\theta_{g}\right)$, which, in combination with $G\left(\theta_{g}\right)$ and crystallographic constraints, provides $D\left(\theta_{d}\right)$.

With the orientation distribution of domains in a ferroelectric ceramics determined from the Taylor estimate, we can predict the effective linear constitutive moduli of ferroelectric ceramics using a self-consistent approach $[10,11]$. The key step in estimating the effective moduli of a heterogeneous ferroelectric is to estimate the average electromechanical fields in the ferroelectric, a procedure requiring the determination of local electromechanical fields in domains, and orientational averaging of local electromechanical fields over all domains and grains. The self-consistent approach is applied to estimate the local electromechanical fields in a domain by an effective medium approximation, where the domain is assumed to be embedded in an infinite homogeneous medium with yet to be determined effective moduli. The electromechanical field in this domain could be determined by Eshelby's equivalent inclusion concept [12], which depends on the effective moduli, and thus usually requires iteration. The Gauss quadrature method [13] is applied to carry out the numerical integration in orientational averaging. The details of numerical implementation can be found in [11].

\section{RESULTS AND DISCUSSION}

\section{Saturation Strain and Polarization}

We now substantiate our theory by a few case studies. We consider generic materials of three distinct crystallographies (tetragonal, rhombohedral, and tetragonalrhombohedral coexistence), evaluate the saturation strain and polarization, and illustrate it for a specific example.

Consider a ferroelectric material such as $\mathrm{BaTiO}_{3}$ that is cubic above the Curie temperature and tetragonal with $\langle 001\rangle_{c}$ polarization at room temperature. If a polycrystal does not have a strong $\langle 001\rangle_{c}$ texture (i.e., if all grains do not have a common pseudocubic axis), the inter-granular constraints are so strong that they prevent any macroscopic saturation strain. Further, the saturation polarization is only a third of single crystal value, compared to previously estimated $83 \%$ without constraint [5]. Specializing to $\mathrm{BaTiO}_{3}$ where the spontaneous polarization is $26 \mu \mathrm{C} / \mathrm{cm}^{2}$ in a single crystal [14], this translates to $8.67 \mu \mathrm{C} / \mathrm{cm}^{2}$, in excellent agreement with experimental observed $8 \mu \mathrm{C} / \mathrm{cm}^{2}$ [14], and in sharp contrast to previous prediction of $21.6 \mu \mathrm{C} / \mathrm{cm}^{2}$. Finally, such a polycrys- 
TABLE 1. Saturation strain and polarization of random ferroelectric polycrystals; $\mathbf{p}^{s}: \mu \mathrm{C} / \mathrm{cm}^{2}$

\begin{tabular}{llll}
\hline & $\mathrm{BaTiO}_{3}(\mathrm{~T})$ & $\mathrm{PZN}-\mathrm{PT}(\mathrm{R})$ & $\mathrm{PZT}(\mathrm{MPB})$ \\
\hline $\mathbf{p}^{s}$ (predicted) & 8.67 & 32.6 & \\
\hline $\mathbf{p}^{s}$ (measured) & $8[14]$ & $35.1[16]$ & \\
\hline $\mathbf{e}^{s}$ (predicted) & 0 & 0 & $0.2 \%$ \\
\hline $\mathbf{e}^{s}$ (measured) & $\approx 0$ & $\mathbf{0 . 0 2 5 \% [ 1 6 ]}$ & $0.18 \%[1]$ \\
\hline
\end{tabular}

tal would be difficult to pole, and will show little macroscopic strain through domain switching. When the polycrystal has $\langle 001\rangle_{c}$ texture, then it can have a saturation strain and polarization almost equal to that of a single crystal. This is consistent with reported saturation polarization as high as $25 \mu \mathrm{C} / \mathrm{cm}^{2}$ in $\mathrm{BaTiO}_{3}$ thin film of $1 \mu \mathrm{m}$ thick [15]. When the film thickness increases to $2 \mu \mathrm{m}$, it drops to about $7 \mu \mathrm{C} / \mathrm{cm}^{2}$. In summary, it is much easier to pole a tetragonal polycrystal with a $\langle 001\rangle_{c}$ texture; this texture is also optimal for large strain actuation.

Rhombohedral systems with $\langle 111\rangle_{c}$ polarization are qualitatively similar. Random rhombohedral polycrystals can not have any saturation strain, and the saturation polarization is only $1 / \sqrt{2}$ of the single crystal value, compared with previous estimated $87 \%$ [5]. This is confirmed in PZN-PT solid solution where saturation strain of $32.6 \mu \mathrm{C} / \mathrm{cm}^{2}$ is predicted in ceramics since the single crystal value is $46.1 \mu \mathrm{C} / \mathrm{cm} 2$ [16], in excellent agreement with experimental measured $35.1 \mu \mathrm{C} / \mathrm{cm}^{2}$ [16]. For the same ceramic, the saturation strain under electric field is measured to be $0.025 \%$, one order of magnitude smaller than that observed in PZT at MPB.

PZT at MPB is the most interesting system from both practical and theoretical points of view. It was originally believed that tetragonal and rhombohedral phases co-exist at this phase boundary, but it was recently recognized that it could actually be a monoclinic phase [17]. Our results predict $0.2 \%$ strain in a soft PZT, in excellent agreement with observed $0.18 \%$ in PZT-5H [1].

All the results on the saturation strain and polarization of different polycrystalline systems are summarized in Table 1.

\section{The Effective Electroelastic Moduli}

Next let us consider the effective electromechanical moduli of a poled piezoelectric ceramic, for example, tetragonal $\mathrm{BaTiO}_{3}$. From the Taylor estimate, the ODF of domains in a poled ferroelectric ceramic composed of tetragonal grains is given by

$$
D\left(\theta_{d}\right)=\frac{1+\cos \left(\theta_{d}\right)}{2},
$$

which in combination with the self-consistent approach yields the effective piezoelectric constants in reasonable agreement with experiment, see Table 2 . The single crystal value is obtained from [18], and experimental data for the ceramic is obtained from [19]. 
TABLE 2. The effective piezoelectric and dielectric constants of poled barium titanate ceramic; $e_{i j}: \mathrm{C} / \mathrm{m}^{2}$

\begin{tabular}{llllll}
\hline & $e_{31}$ & $e_{33}$ & $e_{15}$ & $\kappa_{11} / \kappa^{0}$ & $\kappa_{33} / \kappa^{0}$ \\
\hline estimated & -3.28 & 10.2 & 6.68 & 1604 & 1627 \\
\hline measured & -4.4 & 18.6 & 11.6 & 1266 & 1420 \\
\hline
\end{tabular}

It is worthwhile to notice that the measured elastic moduli in poled $\mathrm{BaTiO}_{3}$ demonstrates very weak anisotropy, despite strong anisotropy in elastic moduli of single crystalline $\mathrm{BaTiO}_{3}$. This suggests that majority of domain switching is 180 degree in nature, which does not change the isotropic elastic symmetry of ceramics. This is consistent with the Taylor estimate. Similar observation could also be made on the dielectric constants, which has even stronger anisotropy in single crystal. The prediction usually overestimates the measured experimental values, which we believe is due to the neglecting of the effects of voids and cracks in ceramics.

\section{Films with Fiber Texture}

We are particularly interested in the effective moduli of piezoelectric polycrystals with fiber texture where all the grains have a common pseudo cubic axis. They have the best polarizability, and thus could offer the best electromechanical coupling. In Figure 1 we present the electromechanical coupling coefficients $k_{31}, k_{33}$, and $k_{15}$ of $\mathrm{BaTiO}_{3}$ single crystals and polycrystals with different textures. 90 degree domains and 180 degree domains exist in ceramics consisting of tetragonal grains. We assume both 90 degree domains and 180 degree domains within all grains are switched to be as closely as possible to the poling direction subjected to the constraint of crystallography in a 'fully poled' ceramics, and only 180 degree domains are switched in a 'partially poled' ceramics. The corresponding ODF for domains, as a result, is uniform for $\boldsymbol{\theta}_{d} \in[0, \pi / 4]$ and $\theta_{d} \in[0, \pi / 2]$, respectively, and zero elsewhere. We notice that in all systems, the polycrystals with $<001>_{c}$ fiber texture have the same $\left(k_{33}\right.$ and $\left.k_{15}\right)$ or higher $\left(k_{31}\right)$ coupling coefficients as single crystals, consistent with the exact relationship derived in [20]. It suggests that fiber texture is also optimal for electromechanical coupling.

\section{CONCLUSIONS}

In summary, we have presented an effective electromechanical theory of polycrystalline ferroelectrics which takes into account both the effect of domains and grains, and gives remarkably good predictions on the polarizability, saturation polarization and strain, the effective texture, and the effective piezoelectric constants of ferroelectric polycrystals. 


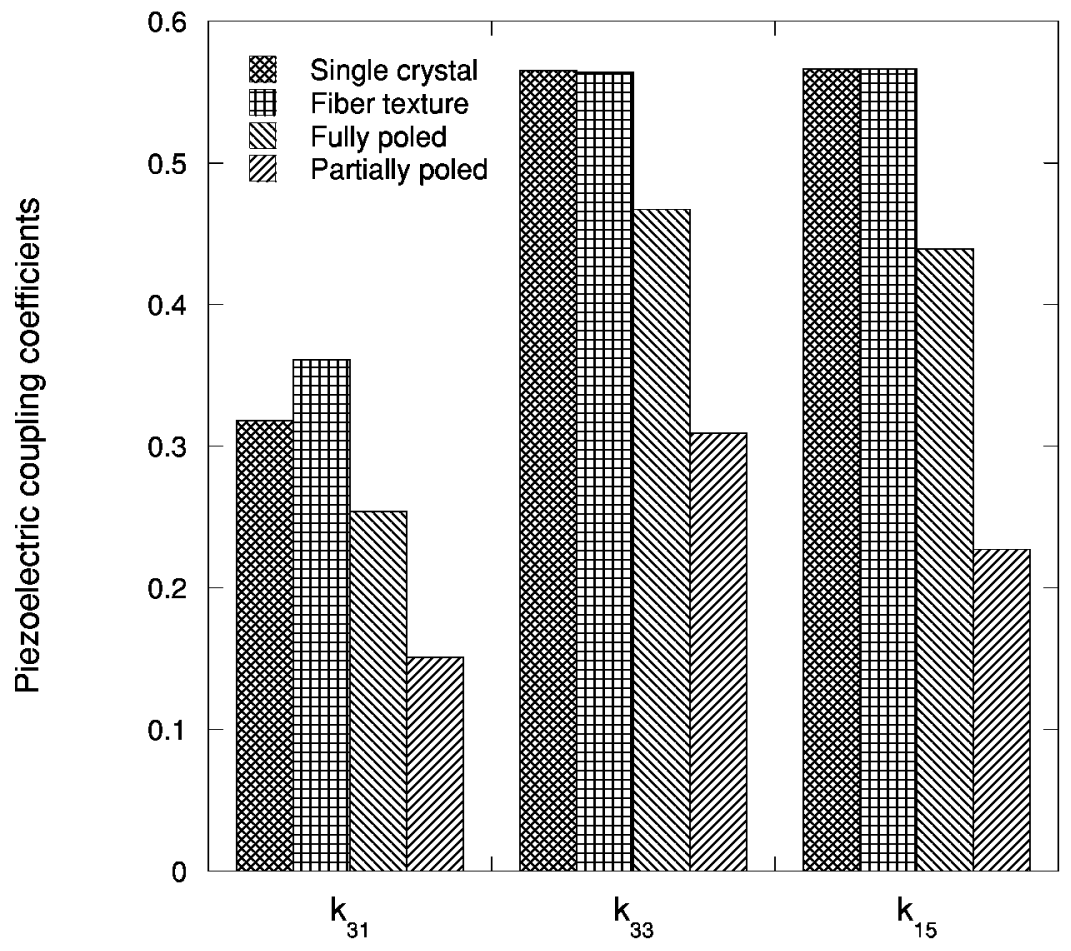

FIGURE 1. The effective electromechanical coupling coefficients of barium titanate ceramics with fiber texture

\section{ACKNOWLEDGMENTS}

The authors gratefully acknowledge the financial support of the Army Research Office through DAAD 19-99-1-0319 and DAAD 19-01-1-0517.

\section{REFERENCES}

1. Shrout, T. R., and Park, S. E., J. Appl. Phys., 82, 1804-1811 (1997).

2. Fu, H., and Cohen, R. E., Nature, 403, 281-2831 (2000).

3. Burcsu, E., Ravichandran, G., and Bhattacharya, K., App. Phys. Lett., 77, 1698-1700 (2000).

4. Shu, Y. C., and Bhattacharya, K., Phil. Mag. B, 81, 2021-2054 (2001).

5. Cross, L. E., "Ferroelectric Ceramics: Tailoring Properties for Specific Application," in Ferroelectric Ceramic, edited by N. Setter and E. L. Colla, Birkhauser Verlag, Berlin, 1993, pp. 1-85.

6. Bhattacharya, K., and Kohn, R. V., Arch. Ration. Mech. An., 139, 99-180 (1997).

7. Li, J. Y., and Bhattacharya, in preparation (2002). 
8. Bhattacharya, K., and Li., J. Y., "Domain Patterns, Texture and Macroscopic Electromechanical Behavior of Ferroelectrics," in Proceedings of The 11th Williamsburg Workshop on Fundamental Physics of Ferroelectrics, edited by H. Krakauer, AIP, 2001, pp. 72-81.

9. DeSimone, A., Arch. Rat. Mech. Anal., 125, 99-143 (1993).

10. Dunn, M. L., J. Appl. Phys., 78, 1533-1541 (1995).

11. Li, J. Y., J. Mech. Phys. Solids, 48, 529-552 (2000).

12. Eshelby, J. D., P. Royal. Soc. London A, 241, 376-396 (1957).

13. Press, W. H., Teukolsky, S. A., W. T., Vetterling, and Flannery, B. P., Numerical Recipes in FORTRAN, 2nd edt., Cambridge University Press, Cambridge, 1992.

14. Jona, F., and Shirane, G., Ferroelectric Crystals, Dover Publications, Ney York, 1993.

15. Jang, J. W., Chung, S. J., Cho, W. J., Hahn, T. S., and Choi, S. S., J Appl. Phys., 81, 6322-6327 (1997).

16. Viehland, D., and Powers, J., J Appl. Phys., 89, 1820-1825 (2001).

17. Noheda, B., Gonzalo, J. A., Cross, L. E., Guo, R., Park, S. E., Cox, D. E., and Shirane, G., Phys. Rev. B., 61, 8687-8695 (2001).

18. Zgonik, M., Bernasconi, P., Duelli, M., Schlesser, R., Günter, P., Garrett, M., Rytz, D., Zhu, Y., and Wu, X., Phys. Rev. B., 50, 5941-5949 (1994).

19. Xu, Y., Ferroelectric Materials and their Application, North-Holland, Amsterdam, 1991.

20. Li, J. Y., Dunn, M. L., and Ledbetter, H., J. Appl. Phys., 86, 4626-4634 (1999). 